1 Hacettepe Journal of Mathematics and Statistics

$\bigcap$ Volume 45 (5) (2016), $1411-1420$

\title{
The existence of extremal solutions to nonlinear fractional integro-differential equations with advanced arguments
}

\author{
Neda Khodabakhshi* ${ }^{* \dagger}$ and S. Mansour Vaezpour ${ }^{\ddagger}$
}

\begin{abstract}
This paper deals with the existence of extremal solutions for nonlinear fractional integro-differential equations with advanced arguments. Our analysis rely on monotone iterative method based on upper and lower solutions. Also, we give an illustrative example in order to indicate the validity of our assumptions.
\end{abstract}

Keywords: Monotone iterative method, Riemann-Liouville fractional derivative, Upper and lower solutions.

2000 AMS Classification: Primary 26A33; Secondary 34K37.

Received: 10.06.2015 Accepted : 11.11.2015 Doi : 10.15672/HJMS.20164514285

\section{Introduction}

Fractional calculus is a branch of mathematical analysis, that provides integrals and derivatives of any arbitrary order and due to their multiple applications in many areas of science and engineering has grown extensively. [1, 3, 4, 7, 8, 9, 10, 11, 14, 15, 16, 17]. The monotone iterative method based on upper and lower solutions is a fruitful tools that provides an efficient mechanism to prove the existence results for nonlinear differential problems. We refer the reader to the book [5] and recent papers [2, 6, 12, 13, 18, 19, 20, $21,22]$.

As far as we know, few authors consider the existence of extremal solutions for nonlinear Riemann-Liouville fractional integro-differential equations with advanced arguments. So this paper is devoted to study of the following nonlinear boundary value problem:

$$
\left\{\begin{array}{l}
\left(D^{\alpha} x(t)\right)^{\prime}=f\left(t, x(t), D^{\alpha} x(t), D^{\beta} x(t), T x(t), S x(t)\right), \quad t \in J:=[0, T], \\
D^{\alpha} x(0)=x^{*},\left.t^{1-\alpha} x(t)\right|_{t=0}=0,0<\beta \leq \alpha \leq 1,
\end{array}\right.
$$

*Department of Mathematics and Computer Sciences, Amirkabir University of Technology, Tehran, Iran. Email: khodabakhshi@aut.ac.ir

${ }^{\dagger}$ Corresponding Author.

$\ddagger_{S}$. Mansour Vaezpour, Email: vaez@aut.ac.ir 
where $f \in C\left(J \times \mathbb{R}^{5}, \mathbb{R}\right)$,

$$
(T x)(t)=\int_{0}^{t} k(t, s) x(s) d s, \quad(S x)(t)=\int_{0}^{T} h(t, s) x(s) d s,
$$

$k(t, s) \in C\left[D, \mathbb{R}^{+}\right], h(t, s) \in C\left[[0, T]^{2}, \mathbb{R}^{+}\right], D=\left\{(t, s) \in \mathbb{R}^{2} \mid 0 \leq s \leq t \leq T\right\}$ and $D^{\alpha}, D^{\beta}$ are the Riemann-Liouville fractional derivatives.

The innovation of this study is that the nonlinear term $f$ involve unknown function $x(t)$ and it's Riemann-Liouville fractional derivatives with different orders and integral operators $T x, S x$. Therefore, from this point of view, we generalize some recent works. Moreover, with a suitable choice of upper and lower solutions and condition on function

$f$, we obtain the existence of extremal solutions and also present iterative sequences which are convergent to them.

This paper is organized as follows: in section 2, some facts and results about fractional calculus are given, also we consider the existence of the extremal solutions for first order nonlinear differential equation, while in spire of [20] we prove the main result in section 3 and we conclude this paper by considering an example in section 4 .

\section{Preliminaries and some lemmas}

In this section, we present some definitions and results which will be needed later.

2.1. Definition. ([4]) The Riemann-Liouville fractional integral of order $\alpha>0$ of a function $f:(0, \infty) \rightarrow \mathbb{R}$ is defined by

$$
I^{\alpha} f(t)=\frac{1}{\Gamma(\alpha)} \int_{0}^{t}(t-s)^{\alpha-1} f(s) d s, \quad t>0,
$$

provided that the right-hand side is pointwise defined.

2.2. Definition. ([4]) The Riemann-Liouville fractional derivative of order $\alpha>0$ of a continuous function $f:(0, \infty) \rightarrow \mathbb{R}$ is defined by

$$
D^{\alpha} f(t)=\frac{1}{\Gamma(n-\alpha)}\left(\frac{d}{d t}\right)^{n} \int_{0}^{t}(t-s)^{n-\alpha-1} f(s) d s \quad t>0,
$$

where $n=[\alpha]+1$, provided that the right-hand side is pointwise defined. In particular, for $\alpha=n, D^{n} f(t)=f^{(n)}(t)$.

1. Remark. The following properties are well known:

$D^{\alpha} I^{\alpha} f(t)=f(t), \alpha>0, f(t) \in L^{1}(0, \infty)$,

$D^{\beta} I^{\alpha} f(t)=I^{\alpha-\beta} f(t), \alpha>\beta>0, f(t) \in L^{1}(0, \infty)$.

2.1. Lemma. ([4]) Let $\operatorname{Re}(\alpha)>0, n=[\operatorname{Re}(\alpha)]+1$ and let $f_{n-\alpha}(t)=I^{n-\alpha} f(t)$ be the fractional integral of order $n-\alpha$. If $f(t) \in L^{1}(0, T)$ and $f_{n-\alpha} \in A C^{n}[0, T]$, then we have the following equality

$$
I^{\alpha} D^{\alpha} f(t)=f(t)-\sum_{i=1}^{n} \frac{f_{n-\alpha}^{(n-i)}(0)}{\Gamma(\alpha-i+1)} t^{\alpha-i} .
$$

2.2. Lemma. The nonlinear fractional differential equation (1.1) is equivalent to the following IVP:

$$
\left\{\begin{array}{l}
u^{\prime}(t)=f\left(t, I^{\alpha} u(t), u(t), I^{\alpha-\beta} u(t), T_{1} u(t), S_{1} u(t)\right), \quad t \in J, \\
u(0)=x^{*}, 0<\beta \leq \alpha \leq 1,
\end{array}\right.
$$


where

$$
\begin{array}{cc}
T_{1} u(t)=\int_{0}^{t} k_{1}(t, s) u(s) d s, & S_{1} u(t)=\int_{0}^{T} h_{1}(t, s) u(s) d s \\
k_{1}(t, s)=\int_{s}^{t} \frac{(\tau-s)^{\alpha-1} k(t, \tau)}{\Gamma(\alpha)} d \tau, & h_{1}(t, s)=\int_{s}^{T} \frac{(\tau-s)^{\alpha-1} h(t, \tau)}{\Gamma(\alpha)} d \tau .
\end{array}
$$

Proof. Take $D^{\alpha} x(t)=u(t)$ in (1.1), taking into account that $\left.t^{1-\alpha} x(t)\right|_{t=0}=0$, we get

$$
x(t)=I^{\alpha} u(t)=\frac{1}{\Gamma(\alpha)} \int_{0}^{t}(t-\tau)^{\alpha-1} u(\tau) d \tau
$$

also

$$
\begin{aligned}
T x(t) & =T\left(I^{\alpha} u(t)\right)=\int_{0}^{t} k(t, s)\left(I^{\alpha} u(t)\right)_{t=s} d s \\
& =\int_{0}^{t} k(t, s)\left(\int_{0}^{s} \frac{(s-\tau)^{\alpha-1} u(\tau)}{\Gamma(\alpha)} d \tau\right) d s \\
& =\int_{0}^{t}\left(\int_{\tau}^{t} \frac{(s-\tau)^{\alpha-1} k(t, s)}{\Gamma(\alpha)} d s\right) u(\tau) d \tau \\
& =\int_{0}^{t}\left(\int_{s}^{t} \frac{(\tau-s)^{\alpha-1} k(t, \tau)}{\Gamma(\alpha)} d \tau\right) u(s) d s \\
& =\int_{0}^{t} k_{1}(t, s) u(s) d s .
\end{aligned}
$$

The same process can be repeated for $S$. So the proof is completed.

Presently, we prove a comparison result for the first order initial value problem (2.1).

2.3. Lemma. Let $w \in C^{1}(J, \mathbb{R})$ satisfy the relations

$$
\left\{\begin{array}{l}
w^{\prime}(t) \geq-K L_{\alpha} w(t)-L w(t)-M L_{\alpha-\beta} w(t)-N T_{1} w(t)-P S_{1} w(t), \\
w(0) \geq 0,0<\beta \leq \alpha \leq 1
\end{array}\right.
$$

where $K, L, M, N, P \geq 0$ are constants and $L_{\alpha} w(t)=\int_{0}^{t} \frac{(t-s)^{\alpha-1} w(s)}{\Gamma(\alpha)} d s$. If

$$
\begin{aligned}
\int_{0}^{T}\left[\frac{K t^{\alpha}}{\Gamma(\alpha+1)}\right. & +L+\frac{M t^{\alpha-\beta}}{\Gamma(\alpha-\beta+1)}+N \int_{0}^{t} k_{1}(t, s) d s \\
& \left.+P \int_{0}^{T} h_{1}(t, s) d s\right] d t<1 .
\end{aligned}
$$

Then $w(t) \geq 0, \forall t \in J$.

Proof. Suppose $w(t) \geq 0$ is not true, then there exists a $t_{0} \in(0, T]$ such that $w\left(t_{0}\right)<0$. Let $\max \left\{w(t): 0 \leq t \leq t_{0}\right\}=\lambda$, then $\lambda \geq 0$.

If $\lambda=0$, the proof is similar to Lemma (2.1) of [20].

If $\lambda>0$, then there exists a $t_{1} \in\left[0, t_{0}\right]$ such that $w\left(t_{1}\right)=\lambda>0$. From (2.2), we have

$$
\begin{aligned}
w^{\prime}(t) \geq & -\lambda\left[\frac{K t^{\alpha}}{\Gamma(\alpha+1)}+L+\frac{M t^{\alpha-\beta}}{\Gamma(\alpha-\beta+1)}\right. \\
& \left.+N \int_{0}^{t} k_{1}(t, s) d s+P \int_{0}^{T} h_{1}(t, s) d s\right], \quad \forall t \in\left[0, t_{0}\right]
\end{aligned}
$$


Thus, we have

$$
\begin{aligned}
& w\left(t_{0}\right)= w\left(t_{1}\right)+\int_{t_{1}}^{t_{0}} w^{\prime}(t) d t \\
& \geq \lambda-\lambda \int_{0}^{T}\left[\frac{K t^{\alpha}}{\Gamma(\alpha+1)}+L+\frac{M t^{\alpha-\beta}}{\Gamma(\alpha-\beta+1)}+N \int_{0}^{t} k_{1}(t, s) d s\right. \\
&\left.\quad+P \int_{0}^{T} h_{1}(t, s) d s\right] d t \\
&=\lambda\left(1-\int_{0}^{T}\left[\frac{K t^{\alpha}}{\Gamma(\alpha+1)}+L+\frac{M t^{\alpha-\beta}}{\Gamma(\alpha-\beta+1)}+N \int_{0}^{t} k_{1}(t, s) d s\right.\right. \\
&\left.\left.\quad+P \int_{0}^{T} h_{1}(t, s) d s\right] d t\right) .
\end{aligned}
$$

Then, by $w\left(t_{0}\right)<0$, we get

$$
\begin{aligned}
\int_{0}^{T} & {\left[\frac{K t^{\alpha}}{\Gamma(\alpha+1)}+L+\frac{M t^{\alpha-\beta}}{\Gamma(\alpha-\beta+1)}+N \int_{0}^{t} k_{1}(t, s) d s\right.} \\
& \left.+P \int_{0}^{T} h_{1}(t, s) d s\right] d t>1
\end{aligned}
$$

which is contradiction.

2.4. Lemma. If (2.3) holds. Then the linear problem

$$
\left\{\begin{array}{l}
u^{\prime}(t)=g(t)-K I^{\alpha} u(t)-L u(t)-M L^{\alpha-\beta} u(t)-N T_{1} u(t)-P S_{1} u(t) \\
u(0)=x^{*}, g \in C(J, \mathbb{R}), 0<\beta \leq \alpha \leq 1
\end{array}\right.
$$

has a unique solution $u^{*} \in C^{1}(J, \mathbb{R})$.

Proof. We know that, $u(t) \in C^{1}(J, \mathbb{R})$ is a solution of $(2.4)$ if and only if $u(t) \in C(J, \mathbb{R})$ is a solution of the following integral equation

$$
\begin{aligned}
u(t) & =x^{*} e^{-\int_{0}^{t} L d s} \\
& +\int_{0}^{t} e^{-\int_{s}^{t} L d \tau}\left(g(s)-K I^{\alpha} u(s)-M I^{\alpha-\beta} u(s)\right. \\
& \left.-N T_{1} u(s)-P S_{1} u(s)\right) d s \\
& =A u(t) .
\end{aligned}
$$


For any $u, v \in C(J, \mathbb{R})$, we show that $A$ is a contraction operator.

$$
\begin{aligned}
|A u(t)-A v(t)| & =\mid \int_{0}^{t} e^{L(s-t)}\left[g(s)-K I^{\alpha} u(s)-M I^{\alpha-\beta} u(s)-N T_{1} u(s)-P S_{1} u(s) d s\right] \\
& -\int_{0}^{t} e^{L(s-t)}\left[g(s)-K I^{\alpha} v(s)-M I^{\alpha-\beta} v(s)-N T_{1} v(s)-P S_{1} v(s)\right] d s \mid \\
& =\mid \int_{0}^{t} e^{L(s-t)}\left[K\left(I^{\alpha}(v-u)(s)\right)+M\left(I^{\alpha-\beta}(v-u)(s)\right)+N\left(T_{1}(v-u)(s)\right)\right. \\
& \left.\leq \int_{0}^{T} \mid K\left(I^{\alpha}(v-u)(s)\right)+M\left(I^{\alpha-\beta}(v-u)(s)\right)+N\left(T_{1}(v-u)(s)\right)\right] d s \mid \\
& \quad+P\left(S_{1}(v-u)(s)\right) \mid d s \\
\leq & \int_{0}^{T}\left[\frac{K s^{\alpha}}{\Gamma(\alpha+1)}+\frac{M s^{\alpha-\beta}}{\Gamma(\alpha-\beta+1)}+N \int_{0}^{t} k_{1}(t, s) d s\right. \\
& \left.+P \int_{0}^{T} h_{1}(t, s) d s\right] d s\|u-v\| .
\end{aligned}
$$

Therefore, by condition (2.3), it follows

$$
\|A u-A v\|<\|u-v\| .
$$

Thus, by Banach contraction principle $A$ has a unique fixed point $u^{*}$, which is unique solution of (2.4).

2.1. Theorem. Let the following assumptions hold:

- $\left(H_{1}\right)$ There exist $u_{0}, v_{0} \in C^{1}(J, \mathbb{R})$ satisfying $u_{0}(t) \leq v_{0}(t), \forall t \in J$,

$$
\left\{\begin{array}{l}
u_{0}^{\prime}(t) \leq f\left(t, I^{\alpha} u_{0}(t), u_{0}(t), I^{\alpha-\beta} u_{0}(t), T_{1} u_{0}(t), S_{1} u_{0}(t)\right), \quad t \in J \\
u_{0}(0) \leq x^{*}, 0<\beta \leq \alpha \leq 1
\end{array}\right.
$$

and $v_{0}$ satisfies inverse inequalities of (2.5).

- $\left(H_{2}\right)$ There exist constants $K, L, M, N, P \geq 0$ which satisfy condition (2.3) and

$$
\begin{aligned}
f(t, x, y, z, v, w)-f(t, \bar{x}, \bar{y}, \bar{z}, \bar{v}, \bar{w}) \geq & -K(x-\bar{x})-L(y-\bar{y})-M(z-\bar{z}) \\
& -N(u-\bar{u})-P(w-\bar{w}) .
\end{aligned}
$$

where $I^{\alpha} u_{0}(t) \leq \bar{x} \leq x \leq I^{\alpha} v_{0}(t), u_{0}(t) \leq \bar{y} \leq y \leq v_{0}(t), I^{\alpha-\beta} u_{0}(t) \leq \bar{z} \leq z \leq$ $I^{\alpha-\beta} v_{0}(t), T_{1} u_{0}(t) \leq \bar{v} \leq v \leq T_{1} v_{0}(t), S_{1} u_{0}(t) \leq \bar{w} \leq w \leq S_{1} v_{0}(t) \forall t \in J$.

Then there exist monotone iterative sequences $\left\{u_{n}\right\},\left\{v_{n}\right\} \subset\left[u_{0}, v_{0}\right]$ which converge uniformly to the extremal solutions $u^{*}, v^{*}$ of (2.1), respectively, where $\left\{u_{n}\right\},\left\{v_{n}\right\}$ are 
defined by

$$
\begin{gathered}
u_{n}(t)=x^{*} e^{-\int_{0}^{t} L d s}+\int_{0}^{t} e^{-\int_{s}^{t} L d \tau}\left[f \left(s, I^{\alpha} u_{n-1}(s), u_{n-1}(s), I^{\alpha-\beta} u_{n-1}(s),\right.\right. \\
\left.T_{1} u_{n-1}(s), S_{1} u_{n-1}(s)\right) \\
-K I^{\alpha}\left(u_{n}-u_{n-1}\right)(s)-L\left(u_{n}-u_{n-1}\right)(s)-M I^{\alpha-\beta}\left(u_{n}-u_{n-1}\right)(s) \\
\left.-N\left(T_{1}\left(u_{n}-u_{n-1}\right)(s)\right)-P\left(S_{1}\left(u_{n}-u_{n-1}\right)(s)\right)\right] d s
\end{gathered}
$$

and

$$
\begin{gathered}
v_{n}(t)=x^{*} e^{-\int_{0}^{t} L d s}+\int_{0}^{t} e^{-\int_{s}^{t} L d \tau}\left[f \left(s, I^{\alpha} v_{n-1}(s), v_{n-1}(s), I^{\alpha-\beta} v_{n-1}(s)\right.\right. \\
\left.T_{1} v_{n-1}(s), S_{1} v_{n-1}(s)\right) \\
-K I^{\alpha}\left(v_{n}-v_{n-1}\right)(s)-L\left(v_{n}-v_{n-1}\right)(s)-M I^{\alpha-\beta}\left(v_{n}-v_{n-1}\right)(s) \\
\left.-N\left(T_{1}\left(v_{n}-v_{n-1}\right)(s)\right)-P\left(S_{1}\left(v_{n}-v_{n-1}\right)(s)\right)\right] d s
\end{gathered}
$$

Also,

$$
u_{0} \leq u_{1} \leq \ldots \leq u_{n} \leq \ldots \leq u^{*} \leq v^{*} \leq \ldots \leq v_{n} \leq \ldots \leq v_{1} \leq v_{0} .
$$

Proof. For $\eta \in\left[u_{0}, v_{0}\right]$, we consider

$$
\left\{\begin{array}{c}
u^{\prime}(t)=g_{\eta}(t)-K I^{\alpha} u(t)-L u(t)-M I^{\alpha-\beta} u(t) \\
\quad-N\left(T_{1} u(t)\right)-P\left(S_{1} u(t)\right) \\
u(0)=x^{*}, 0<\beta \leq \alpha \leq 1
\end{array}\right.
$$

where

$$
\begin{aligned}
g_{\eta}(t) & =f\left(t, I^{\alpha} \eta(t), \eta(t), I^{\alpha-\beta} \eta(t), T_{1} \eta(t), S_{1} \eta(t)\right) \\
& +K I^{\alpha} \eta(t)+L \eta(t)+M I^{\alpha-\beta} \eta(t) \\
& +N\left(T_{1} \eta(t)\right)+P\left(S_{1} \eta(t)\right)
\end{aligned}
$$

By Lemma (2.4), we know (2.6) has a unique solution $u \in C^{1}(J, \mathbb{R})$.

Denote an operator $A:\left[u_{0}, v_{0}\right] \rightarrow C(J, \mathbb{R})$ by $u=A \eta$, then

$$
\begin{aligned}
A \eta & =x^{*} e^{-L t}+\int_{0}^{t} e^{L(s-t)}\left[f\left(s, I^{\alpha} \eta(s), \eta(s), I^{\alpha-\beta} \eta(s), T_{1} \eta(s), S_{1} \eta(s)\right)\right. \\
& +K I^{\alpha} \eta(s)+L \eta(s)+M I^{\alpha-\beta} \eta(s)+N\left(T_{1} \eta(s)\right)+P\left(S_{1} \eta(s)\right) \\
& \left.-K I^{\alpha} u(s)-L u(s)-M I^{\alpha-\beta} u(s)-N\left(T_{1} u(s)\right)-P\left(S_{1} u(s)\right)\right] d s .
\end{aligned}
$$

Now, we show that $u_{0} \leq A u_{0}, A v_{0} \leq v_{0}$ and $A$ is nondecreasing.

For the first claim, let $u_{1}=A u_{0}, p(t)=u_{1}(t)-u_{0}(t)$. we show that $p(t) \geq 0$. By $\left(H_{1}\right)$, we get that

$$
\left\{\begin{aligned}
p^{\prime}(t) \geq & -K I^{\alpha} p(t)-L p(t)-M I^{\alpha-\beta} p(t) \\
& -N\left(T_{1} p(t)\right)-P\left(S_{1} p(t)\right) \\
p(0)= & u_{1}(0)-u_{0}(0)=A u_{0}(0)-u_{0}(0) \geq 0
\end{aligned}\right.
$$

Hence, by Lemma (2.3) $p(t) \geq 0$. Similarly, we can show $A v_{0} \leq v_{0}$.

Now, we show that $A$ is nondecresing. Let $u_{1}=A u_{0}, v_{1}=A v_{0}$ and $p(t)=v_{1}(t)-u_{1}(t)$. 
By $\left(H_{2}\right)$, we have

$$
\left\{\begin{aligned}
p^{\prime}(t) \geq & -K I^{\alpha} p(t)-L(t)-M I^{\alpha-\beta} p(t) \\
& -N\left(T_{1} p(t)\right)-P\left(S_{1} p(t)\right) \\
p(0)= & v_{1}(0)-u_{1}(0)>0
\end{aligned}\right.
$$

So $A$ is nondecreasing.

Next, let $u_{n}=A u_{n-1}, v_{n}=A v_{n-1}, n=1,2, \ldots$. By the properties of the operator $A$, we obtain that

$$
u_{0} \leq u_{1} \leq \ldots \leq u_{n} \leq \ldots \leq u^{*} \leq v^{*} \leq \ldots \leq v_{n} \leq \ldots \leq v_{1} \leq v_{0} .
$$

Clearly, $u_{n}, v_{n}$ satisfy

$$
\begin{aligned}
& \left\{\begin{array}{l}
u_{n}^{\prime}(t)=f\left(t, I^{\alpha} u_{n-1}, u_{n-1}, I^{\alpha-\beta} u_{n-1}, T_{1} u_{n-1}, S_{1} u_{n-1}\right) \\
\quad-K\left(I^{\alpha}\left(u_{n}-u_{n-1}\right)\right)-L\left(u_{n}-u_{n-1}\right)-M\left(I^{\alpha-\beta}\left(u_{n}-u_{n-1}\right)\right) \\
\quad-N\left(T_{1}\left(u_{n}-u_{n-1}\right)\right)-P\left(S_{1}\left(u_{n}-u_{n-1}\right)\right) \\
u_{n}(0)=x^{*}
\end{array}\right. \\
& \left\{\begin{array}{c}
v_{n}^{\prime}(t)=f\left(t, I^{\alpha} v_{n-1}, v_{n-1}, I^{\alpha-\beta} v_{n-1}, T_{1} v_{n-1}, S_{1} v_{n-1}\right) \\
\quad-K\left(I^{\alpha}\left(v_{n}-v_{n-1}\right)\right)-L\left(v_{n}-v_{n-1}\right)-M\left(I^{\alpha-\beta}\left(v_{n}-v_{n-1}\right)\right) \\
\quad-N\left(T_{1}\left(v_{n}-v_{n-1}\right)\right)-P\left(S_{1}\left(v_{n}-v_{n-1}\right)\right) \\
v_{n}(0)=x^{*}
\end{array}\right.
\end{aligned}
$$

The sequences $u_{n}, v_{n}$ are uniformly bounded and equicontinuous, so by Arzela-Ascoli Theorem, we find that $\lim _{n \rightarrow \infty} u_{n}(t)=u^{*}(t)$ and $\lim _{n \rightarrow \infty} v_{n}(t)=v^{*}(t)$ uniformly on $J$, and $u^{*}(t), v^{*}(t)$ are solutions of $(2.1)$.

Finally, we prove that $u^{*}, v^{*}$ are the extremal solutions of $(2.1)$ in $\left[u_{0}, v_{0}\right]$. Let $w \in\left[u_{0}, v_{0}\right]$ be any solution of (2.1), then $A w=w$. By $u_{0} \leq w \leq v_{0}$ and the properties of $A$, we have

$$
u_{n} \leq w \leq v_{n}, \quad n=1,2, \ldots .
$$

Thus, taking limit as $n \rightarrow \infty$, we have $u^{*} \leq w \leq v^{*}$. That is, $u^{*}, v^{*}$ are the extremal solutions of $(2.1)$ in $\left[u_{0}, v_{0}\right]$.

This completes the proof.

\section{Main result}

In this section we prove the existence of extremal solutions of (1.1). Let $C_{1-\alpha}(J, \mathbb{R})=\left\{u \in C(0, T] ; t^{1-\alpha} u \in C(J, \mathbb{R})\right\}$ and $D C_{1-\alpha}(J, \mathbb{R})=\left\{u \in C_{1-\alpha}(J, \mathbb{R}) ; D^{\alpha} u \in C^{1}(J, \mathbb{R})\right\}$.

3.1. Theorem. Assume that:

$\left(H_{1}^{\prime}\right)$ There exist $y_{0}, z_{0} \in D C_{1-\alpha}(J, \mathbb{R})$ such that $y_{0}(t) \leq z_{0}(t)$ and $D^{\alpha} y_{0}(t) \leq D^{\alpha} z_{0}(t)$, are lower and upper solution of (1.1),

$$
\left\{\begin{array}{l}
\left(D^{\alpha} y_{0}(t)\right)^{\prime} \leq f\left(t, y_{0}(t), D^{\alpha} y_{0}(t), D^{\beta} y_{0}(t), T y_{0}, S y_{0}(t)\right) \\
D^{\alpha} y_{0}(0) \leq x^{*},\left.\quad t^{1-\alpha} y_{0}(t)\right|_{t=0}=0
\end{array}\right.
$$

and $z_{0}$ satisfies inverse inequalities of (3.1).

$\left(H_{2}^{\prime}\right)$ There exist constants $K, L, M, N, P \geq 0$ which satisfy condition (2.3) such that

$$
\begin{aligned}
f(t, x, y, z, u, w)-f(t, \bar{x}, \bar{y}, \bar{z}, \bar{u}, \bar{w}) & \geq-K(x-\bar{x})-L(y-\bar{y})-M(z-\bar{z}) \\
& -N(u-\bar{u})-P(w-\bar{w}),
\end{aligned}
$$

where $y_{0}(t) \leq \bar{x} \leq x \leq z_{0}(t), D^{\alpha} y_{0}(t) \leq \bar{y} \leq y \leq D^{\alpha} z_{0}(t), D^{\beta} z_{0}(t) \leq \bar{z} \leq z \leq$ $D^{\beta} z_{0}(t), T y_{0}(t) \leq \bar{u} \leq u \leq T z_{0}(t), S y_{0}(t) \leq \bar{w} \leq w \leq S z_{0}(t)$. 
Then there exist iterative sequences $\left\{y_{n}\right\},\left\{z_{n}\right\}$ which converge uniformly to the extremal solutions $y^{*}, z^{*}$ of (1.1), respectively.

Proof. Let $D^{\alpha} x(t)=u(t)$ in (1.1), then Equation (1.1) is transformed into first order integro-differential equation (2.1). Now, we prove that all the conditions of Theorem (2.1) hold. Let $u_{0}(t)=D^{\alpha} y_{0}(t), v_{0}(t)=D^{\alpha} z_{0}(t)$, we have $u_{0}(t) \leq v_{0}(t)$. Also $y_{0}(t)=$ $I^{\alpha} u_{0}(t), z_{0}(t)=I^{\alpha} v_{0}(t)$, so by $\left(H_{1}^{\prime}\right) u_{0}, v_{0}$ satisfy $\left(H_{1}\right)$. By $\left(H_{2}^{\prime}\right)$, it is easy to see that the condition $\left(\mathrm{H}_{2}\right)$ holds. Therefore, by Theorem (2.1), we obtain that (2.1) has extremal solutions $u^{*}, v^{*} \in C^{1}(J, \mathbb{R})$ in $\left[u_{0}, v_{0}\right]$. Let $y^{*}=I^{\alpha} u^{*}, z^{*}=I^{\alpha} v^{*}$ so it follows that

$$
\left\{\begin{array}{l}
D^{\alpha} y^{*}(t)=u^{*}(t) \\
\left.t^{1-\alpha} y^{*}(t)\right|_{t=0}=0
\end{array}\right.
$$

Since $u^{*}$ satisfies (2.1) and $y^{*}$ satisfies (3.2), then $y^{*}$ is a solution of (1.1). Similarly, we can show that $z^{*}$ is a solution of (1.1). It is easy to show that $y^{*}, z^{*}$ are extremal solutions of (1.1). This completes the proof.

\section{Example}

Consider the following problem:

$$
\left\{\begin{array}{c}
\left(D^{\frac{1}{2}} x(t)\right)^{\prime}=\frac{-1}{10} x(t)-\frac{1+t}{15} D^{\frac{1}{2}} x(t)-\frac{1+t^{2}}{20} D^{\frac{1}{4}} x(t) \\
\quad-\frac{1+t^{3}}{30} \int_{0}^{t} t s x(s) d s-\frac{1+t^{4}}{40} \int_{0}^{1} s x(s) d s, \quad t \in[0,1], \\
D^{\frac{1}{2}} x(0)=0,\left.\quad t^{\frac{1}{2}} x(t)\right|_{t=0}=0
\end{array}\right.
$$

where $\alpha=\frac{1}{2}, \beta=\frac{1}{4}, k(t, s)=t s, h(t, s)=s$. Here,

$$
f(t, x, y, z, u, w)=\frac{-1}{10} x-\frac{1+t}{15} y-\frac{1+t^{2}}{20} z-\frac{1+t^{3}}{30} u-\frac{1+t^{4}}{40} w .
$$

By easy computation, we have

$K=\frac{1}{10}, L=\frac{2}{15}, M=\frac{1}{10}, N=\frac{1}{15}, P=\frac{1}{20}$.

Also,

$$
\begin{aligned}
\int_{0}^{T}\left[\frac{K t^{\alpha}}{\Gamma(\alpha+1)}\right. & +L+\frac{M t^{\alpha-\beta}}{\Gamma(\alpha-\beta+1)}+N \int_{0}^{t} k_{1}(t, s) d s \\
& \left.+P \int_{0}^{T} h_{1}(t, s) d s\right] d t=0.324<1 .
\end{aligned}
$$

Now, take $u_{0}(t)=0, v_{0}(t)=t^{2}$. It is easy to see that $u_{0}, v_{0}$ are lower and upper solution of (4.1). So all the conditions of Theorem (3.1) hold.

Thus there exist iterative sequences $\left\{u_{n}\right\},\left\{v_{n}\right\}$ which converge uniformly to the extremal solutions $u^{*}, v^{*}$ of (4.1), respectively.

Acknowledgement The authors would like to thank the referee for giving useful suggestions and comments for the improvement of this paper.

\section{References}

[1] ASME Journal of Computational and Nonlinear Dynamics, Special Issue "Discontinuous and Fractional Dynamical Systems", Guest Editors: J. A. Tenreiro Machado, Albert Luo, vol. 3, Issue 2, 125 pages, April 2008, ISSN: 1555-1415.

[2] Jankowski, T. Initial value for neutral fractional differential equations involving a RiemannLiouville derivative, Appl. Math. Comput. 219 (2013) 7772-7776.

[3] Journal of Vibration and Control, Sage Pub, Special Issue "Mathematical Methods in Engineering", vol. 13, n. 9-10, pp. 1207-1516, Sept. 2007, ISSN: 1077-5463, Guest Editors: K. Tas, J. A. Tenreiro Machado, Dumitru Baleanu. 
[4] Kilbas, A.A., Srivastava, H. M. and Trujillo, J. J. Theory and applications of fractional differential equations, North-Holland Mathematical Studies, vol. 204, Elsevier Science B.V., Amsterdam, 2006.

[5] Ladde, G.S., Lakshmikantham,V. and Vatsala, A. S. Monotone Iterative Techniques for Nonlinear Differential Equations, Pitman, Boston, 1985.

[6] Liu,Z., Sun, J. and Szanto, I Monotone iterative technique for Riemann-Liouville fractional integro-differential equations with advanced arguments, Results. Math. 63 (2013), 1277-1287.

[7] Miller, K. S., Ross, B. An Introduction to the Fractional Calculus and Differential Equations, Wiley, New York, 1993.

[8] Monje, C. A., Chen, Y., Vinagre, B. M., Xue, D. and Feliu, V. Fractional-Order Systems and Controls: Fundamentals and Applications, Springer, New York, NY, USA, 2010.

[9] Oustaloup, A., Sabatier, J., Lanusse, P., Malti, R., Melchior, P. and Moze, M., An overviewof the CRONE approach in system analysis, modeling and identification, observation and control, in Proceedings of the 17thWorld Congress, International Federation of Automatic Control (IFAC '08), pp. 14254-14265, Soul, Korea, July 2008.

[10] Petras, I. Fractional-Order Nonlinear Systems:Modelling, Analysis and Simulation, Springer, Dordrecht, The Netherlands, 2011.

[11] Podlubny, I. Fractional Differential Equations, Academic Press, San Diego, CA, 1999.

[12] McRae, F. A. Monotone iterative technique and existence results for fractional differential equations, Nonlinear Anal. 71 (2009) 6093-6096.

[13] Ramirez, J. D., Vatsala, A. S.Monotone iterative technique for fractional differential equations with periodic boundary conditions, Opuscula Math. 29 (2009) 289-304.

[14] Tarasov, V. E. Fractional Dynamics: Applications of Fractional Calculus to Dynamics of Particles, Fields and Media, Nonlinear Physical Science. Springer, Beijing, Heidelberg (2011).

[15] Tenreiro Machado, J., Kiryakova, V. and Mainardi, F. A poster about the recent history of fractional calculus, Fract. Calc. Appl. Anal. 13, No 3 (2010), 329-334.

[16] Tenreiro-Machado, J. A., Galhano, A. M. and Trujillo, J. J. Science Metrics on Fractional Calculus Development Since 1966, Fract. Calc. Appl. Anal., 16, No 2 (2013), pp. 479-500.

[17] Uchaikin, V. V. Fractional Derivatives for Physicists and Engineers, Volume I Background and Theory; Volume II Applications, Series: Nonlinear Physical Science, Springer Jointly published with Higher Education Press, 2013.

[18] Wang, G. Monotone iterative technique for boundary value problems of a nonlinear fractional differential equations with deviating arguments, J. Comput. Appl. Math. 236 (2012) $2425-2430$

[19] Wang, G., Agarwal, R. P. and Cabada, A. Existence results and the monotone iterative technique for systems of nonlinear fractional differential equations, Appl. Math. Lett. 25 (2012) 1019-1024.

[20] Wang, G., Baleanu, D. and Zhang, L. Monotone iterative method for a class of nonlinear fractional differential equations, Frac. Calc. Appl. Anal. 15 (2012) 244-252.

[21] Wei, Z., Li, G. and Che, J. Initial value problems for fractional differential equations involving Riemann-Liouville sequential fractional derivative, J. Math. Anal. Appl. 367 (2010) 260-272.

[22] Zhang, S. Monotone iterative method for initial value problem involving Riemann-Liouville fractional derivatives, Nonlinear Anal. 71 (2009) 2087-2093. 
